http://dx.doi.org/10.1590/1678-4162-9298

Arq. Bras. Med. Vet. Zootec., v.69, n.4, p.889-895, 2017

\title{
Sistema de grupos sanguíneos AB em felídeos neotropicais e compatibilidade com gatos domésticos
}

[AB blood group system in neotropical felids and compatibility with domestic cats]

\author{
T.D.P. Silva', M.O. Dreyer ${ }^{2}$, F.P. Back' ${ }^{2}$ L.A. Lacerda ${ }^{2}$, A.D. Damasceno ${ }^{1}$, \\ L.B.M. Araújo ${ }^{1}$, F.J.F. Sant'Ana ${ }^{3 *}$, M.C.S. Fioravanti ${ }^{1}$ \\ ${ }^{1}$ Universidade Federal de Goiás - UFG - Goiânia, GO \\ ${ }^{2}$ Universidade Federal do Rio Grande do Sul - UFRGS - Porto Alegre, RS \\ ${ }^{3}$ Universidade de Brasília - UnB - Brasília, DF
}

\begin{abstract}
RESUMO
O principal sistema de grupos sanguíneos reconhecido para gatos é o $\mathrm{AB}$. Os felinos apresentam anticorpos naturais contra o antígeno do tipo sanguíneo a que não pertencem, o que torna os testes de compatibilidade e as tipagens sanguíneas importantes na prevenção de reações transfusionais. O objetivo deste estudo foi realizar a tipagem sanguínea de oito gatos-mouriscos (Puma yagouaroundi), oito jaguatiricas (Leopardus pardalis), sete gatos-palheiros (Leopardus colocolo), sete gatos domésticos (Felis catus) da raça Persa e oito gatos domésticos sem raça definida (SRD), bem como realizar testes de compatibilidade entre os tipos sanguíneos iguais das diferentes espécies, para avaliar a possibilidade de transfusões interespecíficas. A técnica empregada para a tipagem foi a hemaglutinação em tubos de ensaio. A ocorrência do tipo sanguíneo tipo A foi de $100 \%$ entre as jaguatiricas, os gatos-palheiros e os gatos Persas e de $85,72 \%$ entre os gatos SRD. A ocorrência do tipo B foi de $100 \%$ nos gatos-mouriscos e de $14,28 \%$ nos gatos SRD. Considerando os testes de compatibilidade sanguínea, $87,5 \%(n=4)$ das jaguatiricas foram incompatíveis com os gatos domésticos, $100 \%(n=6)$ dos gatos-palheiros foram compatíveis com os gatos domésticos e $100 \%(n=4)$ dos gatos-mouriscos foram incompatíveis com os gatos domésticos do tipo $\mathrm{B}$.
\end{abstract}

Palavras-chave: Felis colocolo, Leopardus pardalis, Puma yagouaroundi, tipos sanguíneos, xenotransfusão

\begin{abstract}
The blood group system recognized for cats is AB. Antibodies against other blood types occur naturally in cats, which makes the compatibility tests and blood typing important for preventing transfusion reactions. Wild felids need blood transfusions in cases of diseases and when run over on highways. The aim of this study was to perform blood typing of eight jaguarundies (Puma yagouaroundi), eight ocelots (Leopardus pardalis), seven pampas cats (Leopardus colocolo), seven domestic cats (Felis catus) of Persian breed and eight non-pedigree domestic cats (Felis catus), and test compatibility among the different species with the same blood types, to evaluate the possibility of performing interspecific blood transfusions. We conducted the study from August to December. We used haemagglutination in test tubes for typing. The occurrence of blood type A was 100\% among ocelots, pampas cats and domestic cats of Persian breed, while non-pedigree domestic cats showed $85.72 \%$. The occurrence of type B was $100 \%$ for jaguarundis and $14.28 \%$ for non-pedigree domestic cats. Regarding blood compatibility tests, $87.5 \%$ ( $n=$ 4) of the ocelots were incompatible with domestic cats; $100 \%(n=6)$ of the pampas cats were compatible with domestic cats, while $100 \%(n=4)$ of the jaguarundis were incompatible with type B domestic cats.
\end{abstract}

Keywords: blood typing, Felis colocolo, Leopardus pardalis, Puma yagouaroundi, xenotransfusion

Recebido em 5 de novembro de 2016

Aceito em 3 de dezembro de 2016

*Autor para correspondência (corresponding author)

E-mail: santanafjf@yahoo.com 


\section{INTRODUÇÃO}

Os felídeos selvagens são constantes vítimas de atropelamentos em rodovias (Melo e Santos-Filho, 2007), infestações por endo e ectoparasitas (Labruna et al., 2005) e infecções por hemoparasitos, como Babesia spp. (André et al., 2011b) e Candidatus Mycoplasma haemominutum (André et al., 2011a). Transfusões sanguíneas podem ser necessárias e úteis nessas situações, bem como salvar a vida do animal em risco e, assim, colaborar com a preservação da espécie.

Para realização de transfusões sanguíneas seguras e efetivas, são necessários conhecimentos acerca dos grupos sanguíneos (Lanevschi e Wardrop, 2001). O principal sistema sanguíneo descrito para gatos é o $A B$, o qual inclui os tipos $\mathrm{A}, \mathrm{B}$ e o raro tipo $\mathrm{AB}$. Weinstein et al. (2007) demonstraram a ocorrência de um novo tipo sanguíneo, denominado "Mik", no entanto antissoros e reagentes para detectá-lo ainda não estão comercialmente disponíveis. O tipo A é o grupo sanguíneo mundialmente predominante em felinos. $\mathrm{O}$ tipo $\mathrm{AB}$ é encontrado apenas em raças de gatos nas quais há o tipo B em sua população, incluindo gatos domésticos de raças puras específicas (Hohenhaus, 2004). Informações sobre os grupos sanguíneos já estão bem estabelecidas para gatos domésticos, entretanto ainda há poucos estudos relativos aos felinos selvagens. Estudo realizado por Griot-Went e Giger (1999) em 131 felídeos não domésticos pertencentes a 26 espécies distintas, por meio do teste de hemaglutinação em tubos estabelecido para gatos domésticos, mostrou que $80 \%$ dos felídeos apresentaram o tipo A, $18 \%$ o tipo B e $2 \%$ o tipo sanguíneo $\mathrm{AB}$. Quanto à pesquisa de ocorrência de aloanticorpos naturais, o estudo revelou que $90 \%$ do plasma dos felídeos selvagens não reagiram com os tipos $\mathrm{A}$ e $\mathrm{B}$ de outros felídeos do mesmo grupo, sugerindo a não ocorrência de aloanticorpos naturais.

A transfusão sanguínea interespecífica, também chamada de xenotransfusão, é uma técnica que se baseia na transfusão de sangue de um doador para um receptor de espécie diferente. Tal procedimento está sendo reconsiderado, mediante o progresso das pesquisas em xenotransplantação e a necessidade de suprimento sanguíneo (Roux et al., 2007).
Estudo realizado por Silvestre-Ferreira et al. (2006) demonstrou que amostra de soro de gatos selvagens (Felis silvestris) tipo A não aglutinou hemácias de gatos domésticos (Felis catus) do mesmo grupo sanguíneo, fato que, associado aos resultados descritos por Griot-Went e Giger (1999), fundamenta a hipótese de realização de transfusão sanguínea interespecífica, tendo como doador o gato doméstico e receptores às espécies de felídeos selvagens.

$\mathrm{O}$ presente estudo objetivou realizar tipagens sanguíneas para o sistema $\mathrm{AB}$ em felinos selvagens das espécies Puma yagouaroundi, Leopardus pardalis e Leopardus colocolo, bem como em gatos domésticos (Felis catus) da raça Persa e sem raça definida (SRD). Adicionalmente, foram realizados testes de compatibilidade sanguínea entre felinos selvagens e gatos domésticos do mesmo tipo sanguíneo, para se avaliar a possibilidade de realização de transfusões sanguíneas interespecíficas.

\section{MATERIAL E MÉTODOS}

Os felinos selvagens utilizados neste estudo eram criados em cativeiro no Parque Zoológico de Goiânia, exceto quatro gatos-mouriscos, pertencentes ao Centro de Triagem de Animais Silvestres (Cetas), localizado em Goiânia/GO. Os gatos domésticos também eram oriundos do mesmo município. $\mathrm{O}$ trabalho foi realizado após aprovação pelo Comitê de Ética e Pesquisa da Universidade Federal de Goiás, protocolo número 265/2010, e pelo Sistema de Autorização e Informação em Biodiversidade - Sisbio - do Ministério do Meio Ambiente, sob o código de autenticação número 58723968.

Os 38 felídeos utilizados no estudo consistiam de oito gatos-mouriscos ( $P$. yagouaroundi), oito jaguatiricas (L. pardalis), sete gatos-palheiros $(L$. colocolo) e sete gatos domésticos ( $F$. catus) Persa e oito SRD. Para avaliação clínica e coleta de amostras de sangue, os felídeos selvagens e os gatos domésticos foram contidos quimicamente com cetamina (Ketamina, Agener União - Saúde Animal, Brasil) e midazolam (Eurofarma, Brasil) nas doses de $10 \mathrm{mg} / \mathrm{kg}$ e $0,2 \mathrm{mg} / \mathrm{kg}$, respectivamente. As amostras de sangue foram obtidas por meio de punção venosa jugular, utilizando-se tubos para coleta a vácuo (Becton Dinckinson, Brasil) e agulhas 25 x $0,8 \mathrm{~mm}$ 
(Labor Import, Brasil). Foram coletados 3,0mL de sangue de cada animal em tubo contendo EDTA K3 (ácido etilenodiaminotetracético tripotássico) para realização da tipagem sanguínea e teste de compatibilidade. $\mathrm{O}$ sangue foi mantido sob refrigeração a $4^{\circ} \mathrm{C}$ por, no máximo, três dias (Stieger et al., 2005). As amostras foram centrifugadas, e o plasma separado do concentrado de eritrócitos. Os eritrócitos foram submetidos a três lavagens consecutivas com solução salina tamponada $(\mathrm{NaCl}$ 0,9\%), por meio de inversão dos tubos e posterior centrifugação a aproximadamente $1035 \mathrm{~g}$ por cinco minutos, formando uma suspensão de células a 5\%.

A tipagem sanguínea foi realizada por meio do teste de hemaglutinação em tubo de ensaio (Giger et al., 1991; Abrams-Ogg, 2000). Foram colocados em três tubos diferentes, previamente identificados, $50 \mu \mathrm{L}$ de $\mathrm{NaCl} 0,9 \%, 50 \mu \mathrm{L}$ do soro anti-A (soro do felino tipo B) e $50 \mu \mathrm{L}$ de solução anti-B preparada com a lectina do trigo Triticum vulgare (Sigma-Aldrick Corporation, USA), nos quais foram adicionados $25 \mu \mathrm{L}$ da suspensão de eritrócitos a $5 \%$, e, em seguida, eles foram homogeneizados suavemente. Decorridos 15 minutos de incubação em temperatura ambiente, os tubos foram centrifugados por 15 segundos a $1035 \mathrm{~g}$ (Lacerda et al., 2011). A leitura do resultado deu-se pela ressuspensão das células para se observar a presença ou a ausência de aglutinação nos tubos de ensaio. De acordo com Stieger et al. (2005), o grau de aglutinação foi avaliado pelos escores 0 (nenhuma reação: animal negativo para $o$ sorotipo testado), 1+ (vários grumos pequenos em sobrenadante avermelhado: positivo), $2+$ (vários grumos um pouco maiores em sobrenadante ligeiramente avermelhado: positivo), 3+ (um grumo médio e alguns grumos pequenos em sobrenadante quase límpido: positivo) e 4+ (um único grande grumo em sobrenadante límpido: positivo).

$\mathrm{O}$ antissoro anti-A utilizado nos primeiros testes realizados neste experimento foi o soro de um gato do tipo sanguíneo B, previamente tipado no Laboratório de Análises Clínicas Veterinárias (LACVet) da Universidade Federal do Rio Grande do Sul, e a solução de lectina de $T$. vulgare foi a solução anti-B utilizada. Para a confirmação dos tipos $\mathrm{B}$, procedeu-se à repetição da prova, preparando-se uma solução de eritrócitos 5\%, em que $25 \mu \mathrm{L}$ dessa solução foram pipetados em tubo com $50 \mu \mathrm{L}$ de plasma do mesmo animal. Em outro tubo, $25 \mu \mathrm{L}$ da solução de eritrócitos a $5 \%$ de um gato tipo A, já conhecido, foram adicionados a $50 \mu \mathrm{L}$ de plasma do animal a ser testado. Após a confirmação dos tipos B, os soros desses animais foram utilizados como reagentes anti-A para a realização de novos testes de tipagem sanguínea.

O teste de compatibilidade sanguínea entre gatos domésticos e selvagens foi realizado exclusivamente entre as amostras com o mesmo tipo sanguíneo. Foram utilizados os testes de reação cruzada maior, menor e controle, segundo metodologia descrita por Abrams-Ogg (2000). Para cada animal, foram rotulados quatro tubos de ensaio: maior, menor, controle do doador e controle do receptor, para a avaliação a $25^{\circ} \mathrm{C}$, e mais quatro tubos idênticos para avaliação a $37^{\circ} \mathrm{C}$. O resultado da reação foi avaliado investigando-se a presença de hemólise e de aglutinação. Quando não foi possível a visualização de aglutinação macroscopicamente, a solução foi examinada ao microscópio óptico. Os resultados obtidos nos testes de reação cruzada foram submetidos à tabulação $\mathrm{e}$ analisados com o auxílio da estatística descritiva, por meio de determinação das frequências.

\section{RESULTADOS}

Todos os gatos domésticos da raça Persa e 87,5\% dos gatos domésticos SRD foram positivos para o tipo sanguíneo A. Apenas uma fêmea SRD $(12,5 \%)$ foi positiva para o tipo sanguíneo B. Entre as espécies selvagens, $100 \%$ dos gatospalheiros e $100 \%$ das jaguatiricas foram positivos para o tipo A. Entre os gatosmouriscos, $100 \%$ foram positivos para o tipo sanguíneo B (Tab. 1). Não foram encontrados animais positivos para o tipo sanguíneo $\mathrm{AB}$ entre as espécies testadas.

Dois gatos SRD, um gato-palheiro e duas jaguatiricas apresentaram escore de aglutinação $2+$, utilizando-se como reagente anti-A o soro de um gato-mourisco tipo $\mathrm{B}$, no entanto, quando repetida a análise com soro de um gato doméstico tipo $\mathrm{B}$, esses animais passaram a apresentar escore 4+. Os demais animais do tipo sanguíneo A também apresentaram escore de aglutinação igual a 4+, quando se usou o soro de um gato doméstico $\mathrm{B}$ como reagente anti-A 
(Fig. 1A). Todos os animais positivos para o tipo B apresentaram escore de aglutinação $4+$, ao se utilizar a lecitina do $T$. vulgare como reagente anti-B (Fig. 1B).

Tabela 1. Número de animais de cada espécie/raça testados e frequência observada dos tipos sanguíneos segundo o sistema AB

\begin{tabular}{lcccc}
\hline \multirow{2}{*}{ Espécie } & \multirow{2}{*}{$\mathrm{N}$} & \multicolumn{3}{c}{ Tipo sanguíneo (\%) } \\
\cline { 3 - 5 } & & $\mathrm{A}$ & $\mathrm{B}$ & $\mathrm{AB}$ \\
\hline Gato Persa & 7 & 100 & 0 & 0 \\
Gato SRD & 8 & 87,5 & 12,5 & 0 \\
Gato-palheiro & 7 & 100 & 0 & 0 \\
Jaguatirica & 8 & 100 & 0 & 0 \\
Gato-mourisco & 8 & 0 & 100 & 0 \\
\hline
\end{tabular}

Seis testes de compatibilidade sanguínea, realizados entre gatos-palheiros e gatos domésticos da raça Persa e SRD, foram positivos para o tipo sanguíneo A. Todos eles foram compatíveis a $25^{\circ} \mathrm{C}$ e a $37^{\circ} \mathrm{C}$, não demonstrando hemólise nem aglutinação na avaliação macroscópica, mas, em avaliações microscópicas, observou-se que, em três testes, houve a presença de rouleaux a $25^{\circ} \mathrm{C}(2+)$ e, nos outros três testes realizados, observou-se rouleaux a $37^{\circ} \mathrm{C}(1+)$. Cinco testes de compatibilidade foram realizados entre amostras sanguíneas de jaguatiricas e de gatos domésticos da raça Persa e SRD, positivos para o tipo sanguíneo A, porém apenas um teste entre jaguatirica e gato Persa mostrou-se compatível, apresentando rouleaux apenas a $37^{\circ} \mathrm{C}(2+)$. Os testes de compatibilidade sanguínea realizados entre os gatos-mouriscos e o gato doméstico SRD positivo para o tipo B mostraram-se incompatíveis macroscopicamente, com escores variando de $2+$ a $4+$.

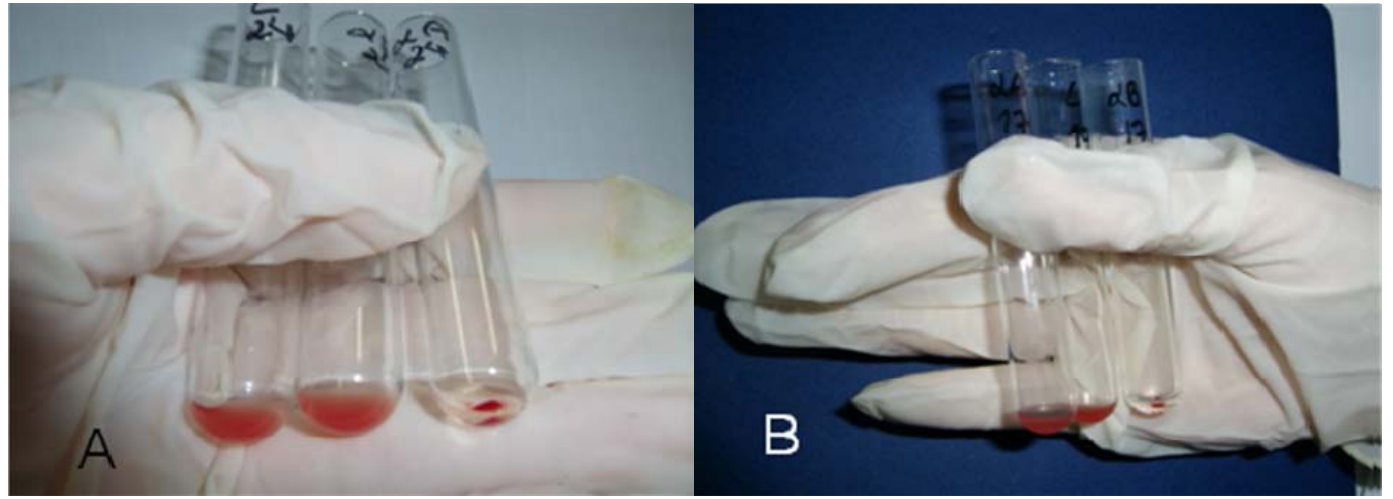

Figura 1. Sistema de grupos sanguíneos AB em felídeos neotropicais e compatibilidade com gatos domésticos. A. Teste de hemaglutinação em tubo de ensaio realizado com amostra sanguínea de jaguatirica (Leopardus pardalis). Da esquerda para a direita, no primeiro tubo, o sangue foi adicionado à solução de $\mathrm{NaCl} 0,9 \%$ (controle). No segundo tubo, testou-se a reação para o grupo sanguíneo tipo B, com adição de solução anti-B. Já no terceiro tubo, foi testada a reação com o grupo A, onde se adicionou soro anti-A. Nota-se reação positiva apenas no terceiro tubo, com escore de aglutinação 4+ para o tipo sanguíneo A. B. Teste de hemaglutinação em tubo de ensaio realizado com amostra sanguínea de gato-mourisco (Puma yagouaroundi). Da esquerda para a direita, no primeiro tubo, testou-se a reação para o grupo sanguíneo tipo A. No segundo tubo, o sangue foi adicionado à solução de $\mathrm{NaCl} 0,9 \%$ (controle). E, no terceiro tubo, foi testada a reação com o grupo $\mathrm{B}$, onde se adicionou soro anti-B. Observa-se se reação positiva apenas no terceiro tubo, com escore de aglutinação 4+ para o tipo sanguíneo B.

\section{DISCUSSÃO}

A distribuição dos grupos sanguíneos do sistema $\mathrm{AB}$ varia com a região geográfica e entre os diferentes tipos de felídeos, mas pouco se sabe sobre os felinos do Brasil (Guerra et al., 2007). Os resultados das tipagens sanguíneas para os gatos domésticos SRD corroboraram os estudos realizados em outros países, que comprovaram ser o tipo A o tipo sanguíneo mais prevalente em felinos (Ejima et al., 1986; Giger et al., 1991; Lacerda et al., 2008; Medeiros et al., 2008). Entretanto, considerando-se o número restrito de animais avaliados neste estudo, a ocorrência de $12,5 \%$ de animais do tipo B pode ser considerada alta. De acordo com Knottenbelt (2002), a determinação da distribuição dos tipos sanguíneos da população felina local pode ajudar a estabelecer o risco de ocorrência de reações transfusionais. No presente estudo, não foram 
encontrados gatos da raça Persa positivos para o tipo B, embora a literatura relate ocorrência variando entre $10 \%$ e $25 \%$ (Brown e Vap, 2006). Em estudo realizado no sul do Brasil, com um número restrito de gatos Persas $(n=20)$, encontrou-se ocorrência de $10 \%$ para o tipo B (Guerra et al., 2007). Entre os felinos selvagens, os resultados aqui encontrados corroboram o estudo realizado por Griot-Went e Giger (1999). Todas as jaguatiricas e os gatos-palheiros foram positivos para o tipo sanguíneo A e todos os gatos-mouriscos foram positivos para o tipo B, o que demonstra que não houve variabilidade genética dentro de nenhuma espécie estudada. As reações ao teste de tipagem sanguínea pela técnica de hemaglutinação em tubos de ensaio foram exatamente iguais às demonstradas para gatos domésticos tanto do tipo A quanto do tipo B.

Todos os testes de reação cruzada entre gatospalheiros e domésticos foram compatíveis, apesar de alguns demonstrarem a presença de rouleaux, o que mostra que transfusões sanguíneas entre as espécies podem ser seguras, não obstante serem necessários estudos para avaliar o comportamento do sangue alogênico in vivo. Segundo Brown e Vap (2006), rouleaux pode estar presente em testes de compatibilidade sanguínea, não estando relacionado à incompatibilidade, que é caracterizada pela presença de aglutinação. Apenas um teste de compatibilidade entre jaguatirica e gato doméstico foi compatível, o que demonstra que mais estudos são necessários para se avaliar a possibilidade de realização de transfusão sanguínea entre essas espécies. Apesar de gatospalheiros e jaguatiricas pertencerem ao mesmo grupo filogenético e apresentarem o mesmo tipo sanguíneo nos testes de tipagem, eles se comportaram de forma diferente quanto aos testes de compatibilidade com gatos domésticos, que pertencem a um grupo filogenético diferente desses (Johnson et al., 2006). Todos os testes entre gatos-palheiros e gatos domésticos foram compatíveis, enquanto apenas um teste entre jaguatirica e gato doméstico foi compatível.

Green et al. (2000), quando pesquisaram anticorpos monoclonais para atuarem como antissoros em testes de tipagem sanguínea para felinos, encontraram resultados que sugerem diferenças entre antígenos eritrocitários $\mathrm{A}$ de alguns animais dos tipos sanguíneos $\mathrm{A}$ e $\mathrm{AB}$.
Essa constatação pode explicar os diferentes comportamentos dos testes de compatibilidade sanguínea dentro do mesmo grupo e dentro da mesma espécie encontrados no presente estudo. Portanto, estudos moleculares são necessários para explicar essas diferenças.

No presente estudo, os testes de compatibilidade sanguínea entre amostras dos gatos-mouriscos, todos do tipo $\mathrm{B}$, e do único gato doméstico positivo para o tipo $\mathrm{B}$ encontrado foram incompatíveis, o que mostra não ser possível a realização de transfusão sanguínea entre as espécies. Mesmo apresentando incompatibilidade em grau 4+ com o sangue de gato doméstico do tipo B, o soro de gatos-mouriscos do tipo B aglutinou eritrócitos do grupo sanguíneo A com intensidade menor do que o soro de gatos domésticos do tipo $\mathrm{B}$, quando utilizado como antissoro anti-A. Tal fato pode estar relacionado a uma menor titularidade de anticorpos, hipótese que pode ser confirmada com a determinação dos títulos de anticorpos, como realizado por Gurkan et al. (2005) e por Lacerda et al. (2011) em gatos SRD para avaliação do risco de reação transfusional na Turquia e em Porto Alegre, Brasil, respectivamente. Além disso, a aglutinação em grau 4+ ocasionada pelo sangue do gato doméstico $\mathrm{B}$ pode ser devido à existência de outro sistema sanguíneo que não foi possível detectar.

No trabalho realizado por Griot-Went e Giger (1999), todos os testes de compatibilidade sanguínea entre animais de grupos filogenéticos diferentes mostraram-se incompatíveis, mas o mesmo teste realizado com animais do mesmo grupo filogenético mostrou $90 \%$ dos resultados compatíveis. Destaca-se que, nesse estudo, animais dos tipos A e B eram utilizados e, mesmo assim, mostravam-se compatíveis e apenas um animal da espécie $F$. silvestris do tipo A foi incompatível com um gato doméstico do tipo B. No entanto, em outro estudo realizado por Silvestre-Ferreira et al. (2006), quando o teste de compatibilidade sanguínea foi realizado entre $F$. silvestris e $F$. catus do tipo A, o resultado foi compatível. Assim, os resultados encontrados no presente estudo diferem dos achados de GriotWent e Giger (1999), pois gatos-palheiros e jaguatiricas, mesmo pertencentes a um grupo filogenético diferente dos gatos domésticos, obtiveram resultados compatíveis quando foi comparado o mesmo tipo sanguíneo (A). 


\section{CONCLUSÕES}

Com base nas condições do presente estudo, pode-se concluir que: 1 - todas as jaguatiricas, todos os gatos-palheiros, todos os gatos Persas e $85,72 \%$ dos gatos SRD pertencem ao tipo sanguíneo A; 2 - todos os gatos-mouriscos e $14,28 \%$ dos gatos SRD apresentam o tipo sanguíneo $\mathrm{B} ; 3$ - o sangue do gato doméstico tipo B não pode ser utilizado para gatosmouriscos (P. yagouaroundi); 4 - o sangue de gatos domésticos (F. catus) é compatível com o sangue de gatos-palheiros (L. colocolo).

\section{REFERÊNCIAS}

ABRAMS-OGG, A.C.G. Practical blood transfusion. In: DAY, M. (Ed.). Manual of canine and feline haematology and transfusion medicine. Hampshire: BSAVA, 2000. p.263-303.

ANDRÉ, M.R.; ADANIA, C.H.; ALLEGRETTI, S.M.; MACHADO, R.Z. Hemoplasmas in wild canids and felids in Brazil. J. Zoo Wildl. Med., v.42, p.342-347, $2011 \mathrm{a}$.

ANDRÉ, M.R.; ADANIA, C.H.; TEIXEIRA, R.H.F. et al. Molecular and sorological detection of Babesia spp. in neotropical and exotic carnivores in Brazilian zoos. J. Zoo Wildl. Med., v.42, p.139-143, 2011 b.

BROWN, D.; VAP, L. Princípios sobre transfusão sanguínea e reação cruzada. In: THRALL, M.A. (Ed.). Hematologia $e$ bioquímica clínica veterinária. São Paulo: Roca, 2006. p.188-198.

EJIMA, H.; KUROKAWA, K.; IKEMOTO, S. Feline red blood cell groups detect by naturally occurring isoantiboby. Jpn. J. Vet. Sci., v.48, p.971-976, 1986.

GIGER, U.; BÜCHELER, J.; PATTERSON, D.F. Frequency and inheritance of A and B blood types in feline breeds of the United States. $J$. Hered., v.82, p.15-20, 1991.

GREEN, J.L.; CHAVEY, P.S.; ANDREWS, G.A.; SMITH, J.E. Production and characterization of murine monoclonal antibodies to feline erythrocyte $\mathrm{A}$ and $\mathrm{B}$ antigens. Comp. Haematol. Int., v.10, p.30-37, 2000 .
GRIOT-WENT, M.E.; GIGER, U. The AB blood group system in wild felids. Anim. Genet., v.30, p.144-147, 1999.

GUERRA, T.A.; LACERDA, L.A.; OLIVEIRA, S.T. et al. Tipagem sanguínea em felinos: 148 gatos domésticos na rotina laboratorial do Lacvet - UFGRGS. Acta Sci. Vet., v.35, p.573-574, 2007.

GURKAN, M.; SEVKET, A.; OZAYTEKIN, E.; DODURKA, T. Titres of alloantibodies against $\mathrm{A}$ and $\mathrm{B}$ blood types in non-pedigree domestic cats in Turkey: assessing the transfusion reaction risk. J. Feline Med. Surg., v.7, p.301-305, 2005.

HOHENHAUS, A.E. Importance of blood groups and blood group antibodies in companion animal. Transfus. Med. Rev., v.18, p.117-126, 2004.

JOHNSON, W.E.; EIZIRIK, E.; PECONSLATTERY, J. The late miocene radiation of modern felidae: a modern assessment. Science, v.311, p.73-76, 2006.

KNOTTENBELT, C.M. The feline AB blood group system and its importance in transfusion medicine. J. Feline Med. Surg., v.4, p.69-76, 2002.

LABRUNA, M.B.; JORGE, R.S.P.; SANA, D.A. Ticks (Acari: Ixodida) on wild carnivores in Brazil. Exp. Appl. Acarol., v.36, p.149-163, 2005.

LACERDA, L.A.; OLIVEIRA, S.T.; GUERRA, T.A. Prevalência dos tipos sanguíneos A, B e AB em gatos domésticos mestiços na cidade de Porto Alegre, Rio Grande do Sul, Brasil. Braz. J. Vet. Res. Anim. Sci., v.45, p.46-53, 2008.

LACERDA, L.A.; OLIVEIRA, S.T.; STEIN, G.G. Titulação de anticorpos anti-a e anti-b em gatos domésticos sem raça definida em Porto Alegre, Rio Grande do Sul, Brasil. Rev. Ceres, v.5, p.51-55, 2011.

LANEVSCHI, A.; WARDROP, K.J. Principles of transfusion medicine in small animals. Can. Vet. J., v.42, p.447-452, 2001.

MEDEIROS, M.A.S.; SOARES, A.M.; ALVIANO, D.S. Frequencies of feline blood types in the Rio de Janeiro area of Brazil. Vet. Clin. Pathol., v.37, p.272-276, 2008. 
MELO, E.S.; SANTOS-FILHO, M. Efeitos da BR-070 na Província Serrana de Cáceres, Mato Grosso, sobre a comunidade de vertebrados silvestres. Rev. Bras. Zoocienc., v.9, p.185-192, 2007.

ROUX, F.A.; SAÏ, P.; DESCHAMPS, J.Y. Xenotransfusions, past and present. Xenotransplantation, v.14, p.208-216, 2007.

SILVESTRE-FERREIRA, A.C.; MARCO, I.; DAUSSA, B. Blood group system in a captive population of european wildcats (Felis silvestris). Vet. Rec., v.159, p.567-568, 2006.
STIEGER, K.; PALOS, H.; GIGER, U. Comparison of various blood-typing methods for the feline AB blood group system. Am. J. Vet. Res., v.66, p.1393-1399, 2005.

WEINSTEIN, N.M.; BLAIS, M.C.; HARRYS, K. Newly recognized blood group in domestic shorthair cats: the mik red cell antigen. J. Vet. Int. Med., v.21, p.287-292, 2007. 